\title{
Early Major Worsening in Ischemic Stroke: Predictors and Outcome
}

\author{
G. Ntaios · D. Lambrou • D. Cuendet • P. Michel
}

Published online: 25 June 2013

(c) Springer Science+Business Media New York 2013

\begin{abstract}
Introduction We aimed to investigate the characteristics and outcome of patients suffering early major worsening (EMW) after acute ischemic stroke (AIS) and assess the parameters associated with it.

Methods All consecutive patients with AIS in the ASTRAL registry until 10/2010 were included. EMW was defined as an NIHSS increase of $\geq 8$ points within the first $24 \mathrm{~h}$ after admission. The Bootstrap version of the Kolmogorov-Smirnov test and the $\chi^{2}$-test were used for the comparison of continuous and categorical covariates, respectively, between patients with and without EMW. Multiple logistic regression analysis was performed to identify independent predictors of EMW.

Results Among 2155 patients, 43 (2.0 \%) had an EMW. EMW was independently associated with hemorrhagic transformation (OR 22.6, $95 \%$ CI 9.4-54.2), cervical artery dissection (OR 9.5, $95 \%$ CI 4.4-20.6), initial dysarthria (OR 3.7, $95 \%$ CI 1.7-8.0), and intravenous thrombolysis (OR 2.1, $95 \%$ CI 1.1-4.3), whereas a negative association was identified with initial eye deviation (OR 0.4, $95 \%$ CI 0.2-0.9). Favorable outcome at 3 and 12 months was less frequent in patients with EMW compared to patients without (11.6 vs. $55.3 \%$ and 16.3 vs. $50.7 \%$, respectively), and case fatality was higher (53.5 vs. $12.9 \%$ and 55.8 vs. $16.8 \%$, respectively). Stroke recurrence within 3 months in surviving patients was similar
\end{abstract}

G. Ntaios $(\bowtie)$

Department of Medicine, University of Thessaly, Biopolis,

41110 Larissa, Greece

e-mail: gntaios@med.uth.gr

G. Ntaios · D. Lambrou - D. Cuendet $\cdot$ P. Michel Neurology Service, Centre Hospitalier Universitaire Vaudois and University of Lausanne, Lausanne, Switzerland between patients with and without EMW (9.3 vs. $9.0 \%$, respectively).

Conclusions Worsening of $\geq 8$ points in the NIHSS score during the first $24 \mathrm{~h}$ in AIS patients is related to cervical artery dissection and hemorrhagic transformation. It justifies urgent repeat parenchymal and arterial imaging. Both conditions may be influenced by targeted interventions in the acute phase of stroke.

Keywords Worsening - Stroke - Acute phase . Cervical artery dissection $\cdot$ Hemorrhagic transformation

\section{Introduction}

Several terms have been used to describe neurological deterioration during the acute phase in patients with ischemic stroke such as "stroke-in-evolution", stroke-inprogress," and "stroke progression". The underlying mechanism may involve either additional brain insults (e.g., ischemic stroke recurrence, hemorrhagic transformation, edema, elevated intracranial pressure, epileptic seizure) or systemic derangements like hypoglycemia, hyponatremia, infection, hypoxemia, and hypotension [1].

Early neurological deterioration is not an uncommon condition: the reported incidence ranges between 26 and $43 \%$ [2-6]. This wide range of estimate is explained by the various definitions used to describe worsening involving different stroke scales, different cut-offs, e.g., increase of the former scale by $\geq 2$ or $\geq 4$ points, and different timeframes, e.g., from $24 \mathrm{~h}$ to 7 days after stroke onset [1]. We were interested in severe, or "major" worsening using a threshold of $\geq 8$ points in the National Institute of Health Stroke (NIHSS) because predictors of milder worsening are already described [6-9], and a higher limit may be 
clinically more meaningful, more specific to identify worsening, and less rater-dependent [1].

Furthermore, limiting this analysis to a narrow time frame when worsening occurs may increase the chances that interventions to correct it may limit its unfavorable effects. To our knowledge, no study has investigated the predictors of early major worsening (EMW) during the acute phase of stroke.

The aim of the present study was to investigate the characteristics and outcome of acute ischemic stroke (AIS) patients suffering an early (within $24 \mathrm{~h}$ ) major (defined as $\geq 8$ point NIHSS increase) worsening and assess the parameters associated with it.

\section{Methods}

All consecutive patients admitted to the stroke unit or intensive care unit of the Centre Hospitalier Universitaire Vaudois (CHUV) between 01/2003 and 10/2010 with a main diagnosis of AIS within $24 \mathrm{~h}$ after last proof of being well were included in the analysis. All patients were prospectively registered in the Acute STroke Registry and Analysis of Lausanne (ASTRAL) [10]; the scientific use of the data registered in ASTRAL was approved by the ethics commission for research on humans of the Canton of Vaud.

The methods applied to collect and register data, and the definitions used in ASTRAL were previously described [10]. In summary, a large range of parameters are prospectively registered: demographics (age, gender, ethnicity), medical history, and cardiovascular risk factors (prestroke modified Rankin score, previous stroke or transient ischemic attack or retinal ischemia, hypertension, diabetes mellitus, dyslipidemia, smoking, atrial fibrillation, coronary artery disease, valvulopathy, low ejection fraction, peripheral artery disease, body-mass-index) current medication (antiplatelets, anticoagulants, antihypertensives, lipid-lowering drugs, hormones, antidiabetics), vital signs (blood pressure, temperature, heart rate), clinical symptoms and examination (i.e., paresis, dysarthria, sensory deficit, visual fields defect, eye deviation, oculomotor brainstem symptoms, cerebellar, ataxic or vestibular signs, aphasia, neglect, level of vigilance), features and severity of the stroke (affected side, vascular territory, brain structures affected, cortical versus subcortical involvement, NIHSS score at admission and at $24 \mathrm{~h}$ ), and treatment (thrombolysis, onset-to-treatment time). Patients who died within $24 \mathrm{~h}$ were assigned a NIHSS of 42 at $24 \mathrm{~h}$. Vital signs (skin temperature, blood pressure, heart rate) and metabolic and hematologic parameters (glucose, creatinine, total cholesterol, white blood cells, hematocrit, platelets) were measured at admission (usually in the emergency room) and at 24-48 h after admission. Findings from acute brain imaging on admission (visibility of the acute ischemic lesion, early hemorrhagic transformation, hyperdense middle cerebral artery sign, chronic stroke lesions, leukoaraiosis, mostly on CT scans) and acute arterial imaging (arterial stenosis $\geq 50 \%$ or occlusion, cervical artery dissection, recanalization status; mostly on CTangiography) were also registered. Stroke pathophysiology was classified according to the TOAST classification [11], but arterial dissections and multiple causes were separately noted. A majority of the patients with initial arterial occlusion also had repeat arterial imaging (CT-angiography, MRangiography, or Doppler) at 24-48 to assess recanalization status. Patients with clinical worsening by $\geq 2$ NIHSS points during the hospital stay usually underwent repeat brain and arterial imaging. Symptomatic intracranial hemorrhage was defined according to ECASS-II [12].

EMW was defined as an increase of the NIHSS score at $24 \mathrm{~h}$ after admission of $\geq 8$ points compared to the NIHSS score at admission. Death within $24 \mathrm{~h}$ was also considered as EMW. Unfavorable clinical outcome was defined as modified Rankin Scale score (mRS) $>2$ at 3 and 12 months and was assessed in the outpatient clinic or on a structured telephone interview by mRS certified personnel. Furthermore, stroke recurrence and mortality were assessed also at 12 months with the same method.

\section{Statistical Analysis}

Associations between the above mentioned clinical and paraclinical variables with EMW were first investigated. Continuous covariates are summarized as median and interquartile range (IQR), and categorical covariates as absolute values and percentage (\%). The bootstrap version of the Kolmogorov-Smirnov test and the $\chi^{2}$-test were used for the comparison of continuous and categorical covariates, respectively, between patients with and without EMW, in the univariate analysis. Backward elimination procedure was applied on the multiple logistic regression model of the complete cases, to determine the significant covariates at $5 \%$ significance level. An imputation process was implemented on the set of the significant independent predictors of EMW (5 imputed datasets), and the final inference was derived by appropriately combining the inferences of the imputed analyses. Statistical analysis was performed with R 2-13-1.

\section{Results}

Among 2209 AIS registered in ASTRAL between January 2003 and October 2010, 54 (2.4\%) were excluded due to missing NIHSS score either at admission or at $24 \mathrm{~h}$ later. Among the remaining 2155 AIS, $43(2.0 \%)$ had an EMW at $24 \mathrm{~h}$ after admission, of whom $23(53.5 \%)$ died within 
3 months. The cause of death was severe ischemic stroke in $12(52.2 \%)$ patients, hemorrhagic transformation in 5 $(21.7 \%)$ patients, post-stroke infection in $3(13.0 \%)$ patients and acute decompensated heart failure in $2(8.7 \%)$ patients. The baseline characteristics of patients with and without EMW are summarized in Table 1. None among patients with lacunar stroke suffered an EMW. Repeat arterial imaging at 24-48 h was performed in $773(35.0 \%)$ patients. There was $1(2.3 \%)$ protocol deviation [i.e., thrombolysis later than $180 \mathrm{~min}$ after stroke onset (or 270 min after 2008)] in the group with early major worsening, and $16(0.8 \%)$ in the group of patients without early major worsening.

In multiple logistic regression analysis, EMW was independently associated with hemorrhagic transformation, cervical artery dissection, admission dysarthria, and intravenous thrombolysis, whereas a negative association was identified with eye deviation on initial examination (Table 2).
Favorable outcome at 3 and 12 months was less frequent in patients with EMW compared to patients without (Table 3). In similar, case fatality at 3 and 12 months were higher in patients with EMW. Stroke recurrence within 3 months was similar between patients with and without EMW.

\section{Discussion}

The present study investigated the predictors and outcome of EMW in a large series of patients with of AIS. We found that hemorrhagic transformation, cervical artery dissection, admission dysarthria, and intravenous thrombolysis were associated with major worsening, whereas there was an inverse association with eye deviation. As expected, unfavorable outcome was more frequent in patients with EMW compared to patients without.

Table 1 Patient characteristics

$N S$ non-significant

${ }^{a}$ Defined as occlusion or $>50 \%$ stenosis

\begin{tabular}{|c|c|c|c|}
\hline & $\begin{array}{l}\text { With early } \\
\text { major worsening } \\
(n=43)\end{array}$ & $\begin{array}{l}\text { Without early } \\
\text { major worsening } \\
(n=2,112)\end{array}$ & $p$ value \\
\hline Age (years) & $67.4 \pm 23.9$ & $72.7 \pm 21.0$ & n.s. \\
\hline Male sex & $19(44.2 \%)$ & $1,187(56.2 \%)$ & n.s. \\
\hline Onset-to-admission time ( $\min )$ & $174 \pm 138$ & $222 \pm 594$ & $<0.01$ \\
\hline NIHSS score at admission & $10.0 \pm 10.0$ & $6.0 \pm 11.0$ & $<0.01$ \\
\hline NIHSS score at $24 \mathrm{~h}$ after admission & $24.0 \pm 15.0$ & $4.0 \pm 9.0$ & $<0.01$ \\
\hline Dysarthria & $33(76.7 \%)$ & $953(45.1 \%)$ & $<0.01$ \\
\hline Eye deviation & $11(25.6 \%)$ & $418(19.8 \%)$ & n.s. \\
\hline Systolic blood pressure at admission $(\mathrm{mmHg})$ & $151.0 \pm 35.0$ & $157.0 \pm 35.5$ & n.s. \\
\hline Subacute systolic blood pressure $(\mathrm{mmHg})$ & $151.0 \pm 25.8$ & $140.0 \pm 27.0$ & $<0.01$ \\
\hline Glucose at admission $(\mathrm{mmol} / \mathrm{l})$ & $6.9 \pm 3.0$ & $6.5 \pm 2.1$ & n.s. \\
\hline Subacute glucose $(\mathrm{mmol} / \mathrm{l})$ & $6.4 \pm 2.5$ & $5.7 \pm 1.6$ & $<0.01$ \\
\hline \multicolumn{4}{|l|}{ Stroke mechanism } \\
\hline Large-artery atherosclerosis & $7(16.3 \%)$ & $282(13.4 \%)$ & n.s. \\
\hline Cardioembolic & $10(23.3 \%)$ & $692(32.8 \%)$ & n.s. \\
\hline Lacunar & $0(0.0 \%)$ & $310(14.7 \%)$ & $<0.05$ \\
\hline Arterial dissection & $10(23.3 \%)$ & $90(4.3 \%)$ & $<0.01$ \\
\hline Multiple/coexisting/other defined & $5(11.6 \%)$ & $189(8.9 \%)$ & n.s. \\
\hline Unknown & $11(25.6 \%)$ & $521(24.7 \%)$ & n.s. \\
\hline \multicolumn{4}{|l|}{ Arterial territory } \\
\hline Anterior circulation & $36(83.7 \%)$ & $1,294(61.3 \%)$ & $<0.01$ \\
\hline Posterior circulation & $6(14.0 \%)$ & $608(28.8 \%)$ & $<0.05$ \\
\hline Acute $\mathrm{CT} / \mathrm{MRI}$ lesion at admission & $23(53.5 \%)$ & $729(34.5 \%)$ & $<0.05$ \\
\hline Significant arterial pathology $\mathrm{y}^{\mathrm{a}}$ & $33(76.7 \%)$ & $968(45.8 \%)$ & $<0.01$ \\
\hline Intravenous thrombolysis & $14(32.6 \%)$ & $355(16.8 \%)$ & $<0.05$ \\
\hline Complete arterial recanalisation & $2(4.7 \%)$ & $147(7.0 \%)$ & n.s. \\
\hline Partial or no arterial recanalisation & $23(53.5 \%)$ & $538(25.5 \%)$ & $<0.01$ \\
\hline Hemorrhagic transformation & $9(20.9 \%)$ & $28(1.3 \%)$ & $<0.01$ \\
\hline Of which thrombolysed/endovascular treatment & $4(44.4 \%)$ & $10(35.7 \%)$ & n.s. \\
\hline
\end{tabular}


Table 2 Predictors of early major worsening in patients with acute ischemic stroke

$O R$ odds-ratio, $C I$ confidence intervals

Table 3 Stroke outcome in patients with and without early major worsening

${ }^{a}$ Defined as modified Rankin Scale score 0-2

\begin{tabular}{lccc}
\hline & OR & $95 \%$ CI & $p$ value \\
\hline Hemorrhagic transformation & 22.6 & $9.4-54.2$ & $<0.0001$ \\
Cervical artery dissection & 9.5 & $4.4-20.6$ & $<0.0001$ \\
Dysarthria & 3.7 & $1.7-8.0$ & $<0.001$ \\
Intravenous thrombolysis & 2.1 & $1.1-4.3$ & 0.03 \\
Eye deviation & 0.4 & $0.2-0.9$ & 0.02 \\
\hline
\end{tabular}

With early

major worsening

$(n=43)$

$\begin{aligned} 2 & (4.6 \%) \\ 5 & (11.6 \%) \\ 7 & (16.3 \%) \\ 23 & (53.5 \%) \\ 24 & (55.8 \%) \\ 4 & (9.3 \%)\end{aligned}$

$2(4.6 \%)$

3-months favorable outcome ${ }^{\mathrm{a}}$

12-months favorable outcome ${ }^{a}$

3-months case fatality

12-months case fatality

Any stroke recurrence within 3 months

(ischemic or hemorrhagic)
Without early major worsening $(n=2,112)$

$15(0.7 \%)$

$1,167(55.3 \%)$

$1,070(50.7 \%)$

$272(12.9 \%)$

$354(16.8 \%)$

$190(9.0 \%)$
Neurologic deterioration in the acute phase of stroke may be caused by additional brain damage (e.g., new stroke, hemorrhagic transformation, brain edema, seizures) or by non-neurological factors (e.g., infection, metabolic derangement like hypo/hyperglycemia and hypo/hypernatremia, hemodynamic insufficiency) [1]. Hence, the knowledge of the parameters which are associated with deterioration is important. Several authors studied the characteristics and outcomes in such patients. The definition of worsening used in these studies varied considerably with regards to the scale, the time frame and the magnitude of deterioration used to define it: Kwan et al. [6] defined early neurological deterioration as an increase of the NIHSS score by $\geq 2$ points (or stroke-related death) between admission and day 5. Leigh et al. [8] defined hyperacute clinical worsening as an increase of the NIHSS by $\geq 4$ points during the first $24 \mathrm{~h}$. The ECASS I group defined early progression as a decrease by $\geq 2$ points of consciousness or motor power or a decrease by $\geq 3$ points of the speech score in the Scandinavian Stroke Scale during the first $24 \mathrm{~h}$ [7]. Tei et al. [9] defined early neurological deterioration as a decrease of $\geq 1$ points in the Canadian Neurological Scale in total, partial anterior or posterior circulation infarcts, or an increase of $\geq 1$ points in the modified Rankin Scale score in lacunar infarcts during the 7 first days after stroke. The advantage of the conservative definitions (i.e., small cut-offs for the increase in the NIHSS scale) is the earlier identification and, potentially, timely treatment of the precipitating factor [1]. On the contrary, more liberal definitions (i.e., larger cut-offs for the increase in the NIHSS scale) are more specific to identify worsening and likely more clinically relevant [1].
The present study differs from previous ones in that the definition of clinical deterioration deliberately uses a high cut-off ( $\geq 8$ points difference in the NIHSS scale).

Several parameters such as blood glucose, diabetes mellitus, blood pressure, body temperature, fibrinogen, and transient ischemic attack were identified as predictors of early neurological deterioration in previous studies [1]. Obviously, the variability of the results between these studies may be attributed to the difference in the definitions used to describe it. In the present study, hemorrhagic transformation was the strongest predictor of EMW. The fact that this parameter remained significant after adjustment for thrombolytic treatment shows that hemorrhagic transformation (either post-thrombolytic, as in $32.6 \%$ of our patients with EMW, or spontaneous) is associated with EMW. It also emphasizes the need for better identification of patients at high risk for hemorrhagic transformation [13], for thrombolytic agents with less hemorrhage risk and avoidance of early anticoagulation [14] .

Cervical artery dissection is also strongly associated with EMW and emphasizes the potential danger of this disorder. Early worsening could be due to the very dynamic changes in acutely dissected cervical arteries, where rapid changes in diameter lumen in the early phase may lead to occlusion and worsening in a subset of patients. These results implicate that urgent parenchymal and arterial imaging should be performed in patients with EMW to identify the presence of any of these two conditions, i.e. hemorrhagic transformation and cervical artery dissection. It may also suggest that arterial imaging should be performed immediately at admission in order to identify patients with cervical artery dissection. An independent association of intravenous thrombolysis with recombinant 
tissue plasminogen activator (rtPA) with EMW was also identified, in addition to hemorrhagic transformation. As a hypothesis to explain this, neurotoxic effects of rtPA [15] may occur in certain individuals, as recently suggested in a retrospective analysis of patients with acute stroke related seizures [16]. Given, the overwhelming evidence of the positive overall effect of thrombolysis in the majority of patients, our findings should not constitute an argument against this treatment in any patient who is eligible for it.

Initial dysarthria was also independently associated with EMW, but eye deviation was inversely associated with EMW. The pathophysiological explanation for these findings remains unclear, but the latter finding was associated with early neurological improvement in two previous reports $[17,18]$. We did not find cerebral edema with mass effect to be associated with EMW, but this may be due to the fact that the time peak of such worsening often occurs more than $24 \mathrm{~h}$ after admission.

The strength of this study is the large number of prospectively registered, consecutive patients with AIS. Also, a wide set of demographic, clinical, laboratory, parenchymal, and arterial imaging parameters were investigated, using pre-specified definitions. Moreover, the delay between stroke onset and admission was short (median of $174 \mathrm{~min}$ ) which means that the NIHSS score measured at admission probably represents a close proximate to the actual NIHSS score at the time of stroke onset. Furthermore, we chose to investigate the predictors of major worsening using a narrow time frame (i.e., $24 \mathrm{~h}$ after admission), which is still early in the course of stroke and may allow for timely therapeutic interventions.

On the limitations side of the study, although the dataset is derived from a prospective stroke registry, the analysis is retrospective, and therefore may be subject to bias. Also, this is a single center study and the conclusions need to be confirmed in other populations. Finally, there may be potential unmeasured confounders such as nosocomial infection; however, this is not expected to be clinically evident so early (i.e., within the first $24 \mathrm{~h}$ after admission).

In conclusion, hemorrhagic transformation and cervical artery dissection are strongly associated with EMW in patients with AIS. In patients with an increase of $\geq 8$ points in the NIHSS score during the first $24 \mathrm{~h}$ after stroke, urgent parenchymal, and arterial imaging should be performed to exclude hemorrhagic transformation and cervical artery dissection.

\section{Conclusion}

Hemorrhagic transformation and cervical artery dissection are strongly associated with EMW in patients with AIS.
Conflict of Interest None.

\section{Reference}

1. Siegler JE, Martin-Schild S. Early neurological deterioration (end) after stroke: the end depends on the definition. Int J Stroke. 2011;6:211-2.

2. Britton M, Roden A. Progression of stroke after arrival at hospital. Stroke. 1985;16:629-32.

3. Davalos A, Cendra E, Teruel J, Martinez M, Genis D. Deteriorating ischemic stroke: risk factors and prognosis. Neurology. 1990;40:1865-9.

4. Jorgensen HS, Nakayama H, Raaschou HO, Olsen TS. Effect of blood pressure and diabetes on stroke in progression. Lancet. 1994:344:156-9.

5. Toni D, Fiorelli M, Gentile M, Bastianello S, Sacchetti ML, Argentino C, et al. Progressing neurological deficit secondary to acute ischemic stroke. a study on predictability, pathogenesis, and prognosis. Arch Neurol. 1995;52:670-5.

6. Kwan J, Hand P. Early neurological deterioration in acute stroke: clinical characteristics and impact on outcome. Quorum J Manag. 2006;99:625-33.

7. Davalos A, Toni D, Iweins F, Lesaffre E, Bastianello S, Castillo J. Neurological deterioration in acute ischemic stroke: potential predictors and associated factors in the European Cooperative Acute Stroke Study (ECASS i). Stroke. 1999;30:2631-6.

8. Leigh R, Zaidat OO, Suri MF, Lynch G, Sundararajan S, Sunshine JL, et al. Predictors of hyperacute clinical worsening in ischemic stroke patients receiving thrombolytic therapy. Stroke. 2004;35:1903-7.

9. Tei H, Uchiyama S, Ohara K, Kobayashi M, Uchiyama Y, Fukuzawa M. Deteriorating ischemic stroke in 4 clinical categories classified by the oxfordshire community stroke project. Stroke. 2000;31:2049-54.

10. Michel P, Odier C, Rutgers M, Reichhart M, Maeder P, Meuli $\mathrm{R}$, et al. The acute stroke registry and analysis of lausanne (astral): design and baseline analysis of an ischemic stroke registry including acute multimodal imaging. Stroke. 2010; 41:2491-8.

11. Adams HP Jr, Bendixen BH, Kappelle LJ, Biller J, Love BB, Gordon DL. Classification of subtype of acute ischemic stroke. Definitions for use in a multicenter clinical trial. Toast. Trial of org 10172 in acute stroke treatment. Stroke. 1993;24:35-41.

12. Hacke W, Kaste M, Fieschi C, von Kummer R, Davalos A, Meier $\mathrm{D}$, et al. Randomised double-blind placebo-controlled trial of thrombolytic therapy with intravenous alteplase in acute ischaemic stroke (ECASS ii). Second European-Australasian acute stroke study investigators. Lancet. 1998;352:1245-51.

13. Strbian D, Engelter S, Michel P, Meretoja A, Sekoranja L, Ahlhelm FJ, et al. Symptomatic intracranial hemorrhage after stroke thrombolysis: the sedan score. Ann Neurol. 2012;71:634-41.

14. Paciaroni M, Agnelli G, Micheli S, Caso V. Efficacy and safety of anticoagulant treatment in acute cardioembolic stroke: a meta-analysis of randomized controlled trials. Stroke. 2007;38: 423-30.

15. Yepes M, Roussel BD, Ali C, Vivien D. Tissue-type plasminogen activator in the ischemic brain: more than a thrombolytic. Trends Neurosci. 2009;32:48-55.

16. Alvarez V, Rossetti AO, Papavasileiou V, Michel P. Acute seizures in acute ischemic stroke: Does thrombolysis have a role to play? J Neurol. 2012. 
17. Felberg RA, Okon NJ, El-Mitwalli A, Burgin WS, Grotta JC, Alexandrov AV. Early dramatic recovery during intravenous tissue plasminogen activator infusion: clinical pattern and outcome in acute middle cerebral artery stroke. Stroke. 2002;33:1301-7.
18. Mikulik R, Dusek L, Hill MD, Fulep E, Grotta JC, Ribo M, et al. Pattern of response of national institutes of health stroke scale components to early recanalization in the clotbust trial. Stroke. 2010;41:466-70. 